International Journal of Pure and Applied Mathematics

Volume 103 No. 4 2015, 653-666

ISSN: 1311-8080 (printed version); ISSN: 1314-3395 (on-line version)

url: http://www.ijpam.eu

doi: http://dx.doi.org/10.12732/ijpam.v103i4.5

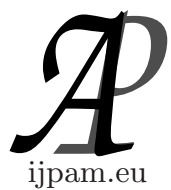

\title{
BIOPERATIONS ON $\alpha$-OPEN SETS IN TOPOLOGICAL SPACES
}

\author{
Alias B. Khalaf ${ }^{1}$, Saeid Jafari ${ }^{2}$, Hariwan Z. Ibrahim ${ }^{3}$ \\ ${ }^{1}$ Department of Mathematics \\ Faculty of Science \\ University of Duhok \\ Kurdistan, Region, IRAQ \\ ${ }^{2}$ College of Vestsjaelland South \\ Herrestraede 11, 4200 Slagelse, DENMARK \\ ${ }^{3}$ Department of Mathematics \\ Faculty of Science \\ University of Zakho \\ Kurdistan, Region, IRAQ
}

Abstract: In this paper, we introduce the concept of $\alpha_{\left[\gamma, \gamma^{\prime}\right]}$-open sets in topological spaces and study some of their properties. Furthermore, we offer a new class of functions called $\left(\alpha_{\left[\gamma, \gamma^{\prime}\right]}, \alpha_{\left[\beta, \beta^{\prime}\right]}\right)$-continuous functions and investigate their fundamental properties.

AMS Subject Classification: 54A05, 54A10

Key Words: bioperation, $\alpha$-open set, $\alpha_{\left[\gamma, \gamma^{\prime}\right]}$-open set

\section{Introduction}

Njastad [3] introduced $\alpha$-open sets in a topological space and studied some of its properties. Kasahara [2] defined the concept of an operation on topological spaces and introduced $\alpha$-closed graphs of an operation. Ogata [4] called the operation $\alpha$ as $\gamma$ operation and introduced the notion of $\tau_{\gamma}$ which is the collection

Received: April 3, 2015

(C) 2015 Academic Publications, Ltd.

$\S$ Correspondence author url: www.acadpubl.eu 
of all $\gamma$-open sets in a topological space $(X, \tau)$. H. Z. Ibrahim [6] defined the concept of an operation on $\alpha O(X, \tau)$ and introduced $\alpha_{\gamma}$-open sets in topological spaces and studied some of their basic properties. In this paper, we have introduced and studied the notion of $\alpha O(X, \tau)_{\left[\gamma, \gamma^{\prime}\right]}$ which is the collection of all

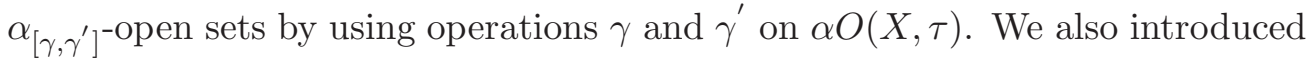
the class of $\left(\alpha_{\left[\gamma, \gamma^{\prime}\right]}, \alpha_{\left[\beta, \beta^{\prime}\right]}\right)$-continuous functions and investogated some of its important properties.

\section{Preliminaries}

Throughout this paper, $(X, \tau)$ and $(Y, \sigma)$ represent non-empty topological spaces on which no separation axioms are assumed, unless otherwise mentioned. Let $(X, \tau)$ be a topological space and $A$ a subset of $X$. The closure of $A$ and the interior of $A$ are denoted by $C l(A)$ and $\operatorname{Int}(A)$, respectively. A subset $A$ of a topological space $(X, \tau)$ is said to be $\alpha$-open [3] if $A \subseteq \operatorname{Int}(C l(\operatorname{Int}(A)))$. The complement of an $\alpha$-open set is said to be $\alpha$-closed. The intersection of all $\alpha$ closed sets containing $A$ is called the $\alpha$-closure of $A$ and is denoted by $\alpha C l(A)$. The family of all $\alpha$-open (resp. $\alpha$-closed) sets in a topological space $(X, \tau)$ is denoted by $\alpha O(X, \tau)$ (resp. $\alpha C(X, \tau)$ ). An operation $\gamma[2]$ on a topology $\tau$ is a mapping from $\tau$ into power set $P(X)$ of $X$ such that $V \subseteq V^{\gamma}$ for each $V \in \tau$, where $V^{\gamma}$ denotes the value of $\gamma$ at $V$. A subset $A$ of $X$ with an operation $\gamma$ on $\tau$ is called $\gamma$-open [4] if for each $x \in A$, there exists an open set $U$ of $X$ containing $x$ such that $U^{\gamma} \subseteq A$. Clearly $\tau_{\gamma} \subseteq \tau$. Complements of $\gamma$-open sets are called $\gamma$-closed. An operation $\gamma: \alpha O(X, \tau) \rightarrow P(X)[6]$ is a mapping satisfying the condition, $V \subseteq V^{\gamma}$ for each $V \in \alpha O(X, \tau)$. We call the mapping $\gamma$ an operation on $\alpha O(X, \tau)$. A subset $A$ of $X$ is called an $\alpha_{\gamma}$-open set [6] if for each point $x \in A$, there exists an $\alpha$-open set $U$ of $X$ containing $x$ such that $U^{\gamma} \subseteq A$. We denote the set of all $\alpha_{\gamma}$-open sets of $(X, \tau)$ by $\alpha O(X, \tau)_{\gamma}$. An operation $\gamma$ on $\alpha O(X, \tau)$ is said to be $\alpha$-regular [6] if for every $\alpha$-open sets $U$ and $V$ containing $x \in X$, there exists an $\alpha$-open set $W$ of $x$ such that $W^{\gamma} \subseteq U^{\gamma} \cap V^{\gamma}$. An operation $\gamma$ on $\alpha O(X, \tau)$ is said to be $\alpha$-open [6] if for every $\alpha$-open set $U$ of $x \in X$, there, exists an $\alpha_{\gamma}$-open set $V$ of $X$ such that $x \in V$ and $V \subseteq U^{\gamma}$.

\section{3. $\alpha_{\left[\gamma, \gamma^{\prime}\right]}$-Open Sets}

Definition 3.1. A non-empty subset $A$ of $(X, \tau)$ is said to be $\alpha_{\left[\gamma, \gamma^{\prime}\right]^{-o p e n}}$ if for each $x \in A$ there exist $\alpha$-open sets $U$ and $V$ of $X$ containing $x$ such 
that $U^{\gamma} \cap V^{\gamma^{\prime}} \subseteq A$. The set of all $\alpha_{\left[\gamma, \gamma^{\prime}\right]^{-o p e n}}$ sets of $(X, \tau)$ is denoted by $\alpha O(X, \tau)_{\left[\gamma, \gamma^{\prime}\right]}$. We suppose that the empty set is $\alpha_{\left[\gamma, \gamma^{\prime}\right]^{-}}$open for any operations $\gamma$ and $\gamma^{\prime}$.

Proposition 3.2. If $A_{i}$ is $\alpha_{\left[\gamma, \gamma^{\prime}\right]}$-open for every $i \in I$, then $\cup\left\{A_{i}: i \in I\right\}$ is $\alpha_{\left[\gamma, \gamma^{\prime}\right]}$-open.

Proof. Let $x \in \cup_{i \in I} A_{i}$, then $x \in A_{i}$ for some $i \in I$. Since $A_{i}$ is an $\alpha_{\left[\gamma, \gamma^{\prime}\right]^{-}}$ open set, so there exist $\alpha$-open sets $U$ and $V$ of $(X, \tau)$ such that $x \in U \cap V \subseteq$ $U^{\gamma} \cap V^{\gamma^{\prime}} \subseteq A_{i} \subseteq \cup_{i \in I} A_{i}$. Therefore $\cup_{i \in I} A_{i}$ is an $\alpha_{\left[\gamma, \gamma^{\prime}\right]^{-}}$open set of $(X, \tau)$.

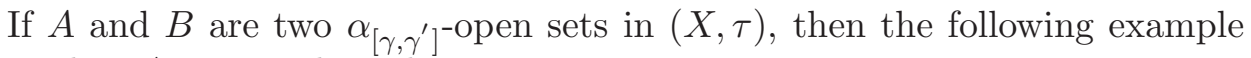

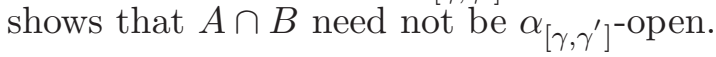

Example 3.3. Let $X=\{a, b, c\}$ and $\tau=\{\phi, X,\{b\},\{c\},\{a, b\},\{b, c\}\}$ be a topology on $X$. For each $A \in \alpha O(X, \tau)$, we define two operations $\gamma$ and $\gamma^{\prime}$, by

$$
A^{\gamma}= \begin{cases}C l(A) & \text { if } c \in A, \\ X & \text { if } c \notin A,\end{cases}
$$

and

$$
A^{\gamma^{\prime}}= \begin{cases}A & \text { if } A \neq\{b\} \\ X & \text { if } A=\{b\} .\end{cases}
$$

Then, it is obvious that the sets $\{a, b\}$ and $\{b, c\}$ are $\alpha_{\left[\gamma, \gamma^{\prime}\right]}$-open, however their intersection $\{b\}$ is not $\alpha_{\left[\gamma, \gamma^{\prime}\right]}$-open.

A subfamily $\tau$ of the power set $P(X)$ of a non-empty set $X$ is called a supratopology [1] on $X$ if $\tau$ satisfies the following conditions:

1. $\tau$ contains $\phi$ and $X$,

2. $\tau$ is closed under the arbitrary union.

The pair $(X, \tau)$ is called a supratopological space.

From the above example we notice that the family of all $\alpha_{\left[\gamma, \gamma^{\prime}\right]}$-open subsets of a space $X$ is a supratopology and need not be a topology in general.

In the following proposition, the intersection of two $\alpha_{\left[\gamma, \gamma^{\prime}\right]}$-open sets is also $\alpha_{\left[\gamma, \gamma^{\prime}\right]}$-open.

Proposition 3.4. Let $\gamma$ and $\gamma^{\prime}$ be $\alpha$-regular operations. If $A$ and $B$ are $\alpha_{\left[\gamma, \gamma^{\prime}\right]}$-open, then $A \cap B$ is $\alpha_{\left[\gamma, \gamma^{\prime}\right]^{-o p e n}}$.

Proof. Let $x \in A \cap B$, then $x \in A$ and $x \in B$. Since $A$ and $B$ are $\alpha_{\left[\gamma, \gamma^{\prime}\right]^{-}}$

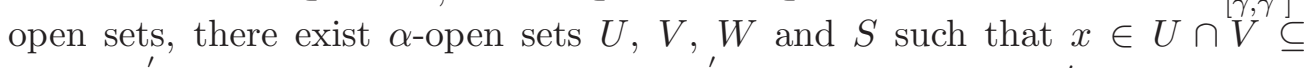
$U^{\gamma} \cap V^{\gamma^{\prime}} \subseteq A$ and $x \in W \cap S \subseteq W^{\gamma} \cap S^{\gamma^{\prime}} \subseteq B$. Since $\gamma$ and $\gamma^{\prime}$ are $\alpha$-regular 
operations, then there exist an $\alpha$-open sets $K$ and $L$ containing $x$ such that $K^{\gamma} \cap L^{\gamma^{\prime}} \subseteq\left(U^{\gamma} \cap W^{\gamma}\right) \cap\left(V^{\gamma^{\prime}} \cap S^{\gamma^{\prime}}\right)=\left(U^{\gamma} \cap V^{\gamma^{\prime}}\right) \cap\left(W^{\gamma} \cap S^{\gamma^{\prime}}\right) \subseteq A \cap B$. This implies that $A \cap B$ is $\alpha_{\left[\gamma, \gamma^{\prime}\right]^{-o p e n ~ s e t . ~}}$

Remark 3.5. By the above propositon, if $\gamma$ and $\gamma^{\prime}$ are $\alpha$-regular operations, then $\alpha O(X, \tau)_{\left[\gamma, \gamma^{\prime}\right]}$ forms a topology on $X$.

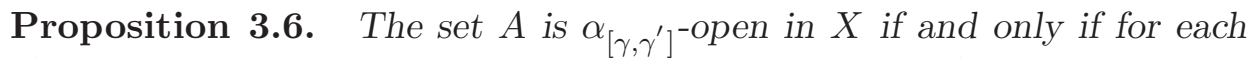
$x \in A$, there exists an $\alpha_{\left[\gamma, \gamma^{\prime}\right]^{-}}$open set $B$ such that $x \in B \subseteq A$.

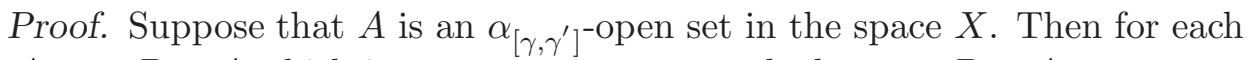

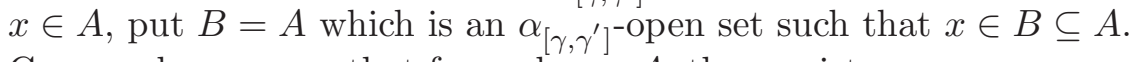

Conversely, suppose that for each $x \in A$, there exists an $\alpha_{\left[\gamma, \gamma^{\prime}\right]^{-}}$open set $B$ such that $x \in B \subseteq A$. Thus $A=\cup_{x \in A} B_{x}$, where $B_{x} \in \alpha O(X, \tau)_{\left[\gamma, \gamma^{\prime}\right]}$. Therefore, $A$ is an $\alpha_{\left[\gamma, \gamma^{\prime}\right]}$-open set.

Proposition 3.7. If $A$ is $\alpha_{\left[\gamma, \gamma^{\prime}\right]}$-open, then $A$ is $\alpha$-open.

The converse of the above proposition need not be true in general as it is shown below.

Example 3.8. Let $(X, \tau), \gamma$ and $\gamma^{\prime}$ be the same space and the same operations as in Example 3.3. Then $\{b\}$ is $\alpha$-open but not $\alpha_{\left[\gamma, \gamma^{\prime}\right]}$-open.

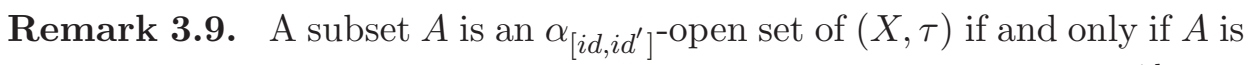
$\alpha$-open in $(X, \tau)$. The operation $i d: \alpha O(X, \tau) \rightarrow P(X)$ is defined by $V^{i d}=V$ for any set $V \in \alpha O(X, \tau)$. This operation is called the identity operation on $\alpha O(X, \tau)$. Therefore $\alpha O(X, \tau)_{\left[i d, i d^{\prime}\right]}=\alpha O(X, \tau)$.

Remark 3.10. From Remark 3.9 and [[6]; Remark 2.3] we have $\alpha O(X, \tau)_{\left[i d, i d^{\prime}\right]}=$ $\alpha O(X, \tau)=\alpha O(X, \tau)_{i d}=\alpha O(X, \tau)_{i d^{\prime}}$.

Remark 3.11. The following example shows that the concept of $\alpha_{\left[\gamma, \gamma^{\prime}\right]^{-}}$ open and open are independent.

Example 3.12. Let $X=\{a, b, c\}$ and $\tau=\{\phi, X,\{a\}\}$ be a topology on $X$. For each $A \in \alpha O(X, \tau)$ we define two operations $\gamma$ and $\gamma^{\prime}$, respectively, by $A^{\gamma}=X$ and

$$
A^{\gamma^{\prime}}= \begin{cases}A & \text { if } A=\{a, b\} \\ X & \text { if } A \neq\{a, b\}\end{cases}
$$

Then, $\alpha_{\left[\gamma, \gamma^{\prime}\right]}$-open sets are $\phi, X$, and $\{a, b\}$. 
Definition 3.13. [5] A non-empty subset $A$ of $(X, \tau)$ is said to be $\left[\gamma, \gamma^{\prime}\right]$ open if for each $x \in A$ there exist open sets $U$ and $V$ of $X$ containing $x$ such that $U^{\gamma} \cap V^{\gamma^{\prime}} \subseteq A$.

Proposition 3.14. If $A$ is $\left[\gamma, \gamma^{\prime}\right]$-open, then $A$ is $\alpha_{\left[\gamma, \gamma^{\prime}\right]}$-open.

The converse of the above proposition need not be true in general as it is shown below.

Example 3.15. Let $X=\{a, b, c\}$ and $\tau=\{\phi, X,\{b\}\}$ be a topology on $X$. For each $A \in \alpha O(X, \tau)$, we define two operations $\gamma$ and $\gamma^{\prime}$, respectively, by $A^{\gamma}=A^{\gamma^{\prime}}=A$. Then $\{a, b\}$ is $\alpha_{\left[\gamma, \gamma^{\prime}\right]^{-o p e n}}$ but not $\left[\gamma, \gamma^{\prime}\right]$-open.

Remark 3.16. If $A$ is $\left(\gamma, \gamma^{\prime}\right)$-open [7], then $A$ is $\alpha_{\left[\gamma, \gamma^{\prime}\right]^{-o p e n . ~}}$

Proposition 3.17. If $A$ is $\alpha_{\gamma^{-o p e n}}$ and $B$ is $\alpha_{\gamma^{\prime}}$-open, then $A \cap B$ is $\alpha_{\left[\gamma, \gamma^{\prime}\right]}$-open.

Proposition 3.18. If $A$ is $\alpha_{\gamma}$-open, then $A$ is $\alpha_{\left[\gamma, \gamma^{\prime}\right]}$-open for any operation $\gamma^{\prime}$.

The converse of Proposition 3.18 need not be true in general as it is shown below.

Example 3.19. Let $X=\{a, b, c\}$ and $\tau$ be a discrete topology on $X$. For each $A \in \alpha O(X, \tau)$ we define two operations $\gamma$ and $\gamma^{\prime}$, respectively, by

$$
A^{\gamma}=A^{\gamma^{\prime}}= \begin{cases}A & \text { if } A=\{a, b\} \text { or }\{b, c\} \\ X & \text { otherwise. }\end{cases}
$$

Then $\{b\}$ is $\alpha_{\left[\gamma, \gamma^{\prime}\right]^{\prime}}$ open but not $\alpha_{\gamma^{-}}$open.

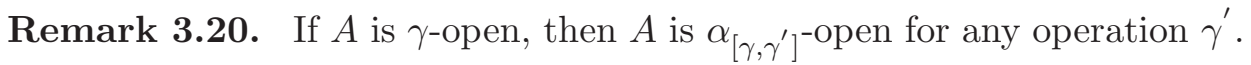

Proposition 3.21. Let $X: \alpha O(X, \tau) \rightarrow P(X)$ be an operation defined by $U^{X}=X$ for every $U \in \alpha O(X, \tau)$. Then $A$ is $\alpha_{\gamma}$-open if and only if $A$ is $\alpha_{[\gamma, X]^{-o p e n}}$.

Definition 3.22. A topological space $(X, \tau)$ is said to be $\alpha_{\gamma}$-regular if for each $x \in X$ and for each $\alpha$-open set $V$ in $X$ containing $x$, there exists an $\alpha$-open set $U$ in $X$ containing $x$ such that $U^{\gamma} \subseteq V$.

In the following proposition, we give a condition under which the family of $\alpha$-open sets is identical to the family of $\alpha_{\gamma}$-open sets.

Proposition 3.23. A topological space $(X, \tau)$ with an operation $\gamma$ on $\alpha O(X, \tau)$ is $\alpha_{\gamma}$-regular if and only if $\alpha O(X, \tau)=\alpha O(X, \tau)_{\gamma}$. 
Remark 3.24. If a topological space $(X, \tau)$ is $\alpha_{\gamma}$-regular, then $\tau \subseteq$ $\alpha O(X, \tau)_{\gamma}$.

Definition 3.25. A topological space $(X, \tau)$ is said to be $\alpha_{\left[\gamma, \gamma^{\prime}\right]}$-regular if for each point $x$ in $X$ and every $\alpha$-open set $U$ containing $x$ there exist $\alpha$-open sets $W$ and $S$ of $x$ such that $W^{\gamma} \cap S^{\gamma^{\prime}} \subseteq U$.

Proposition 3.26. A topological space $(X, \tau)$ with operations $\gamma$ and $\gamma^{\prime}$ on $\alpha O(X, \tau)$ is $\alpha_{\left[\gamma, \gamma^{\prime}\right]}$-regular if and only if $\alpha O(X, \tau)=\alpha O(X, \tau)_{\left[\gamma, \gamma^{\prime}\right]}$.

Proof. Let $(X, \tau)$ be $\alpha_{\left[\gamma, \gamma^{\prime}\right]}$-regular and $A \in \alpha O(X, \tau)$. Since $(X, \tau)$ is $\alpha_{\left[\gamma, \gamma^{\prime}\right]}$-regular, then for each $x \in A$, there exist $\alpha$-open sets $W$ and $S$ of $x$ such that $W^{\gamma} \cap S^{\gamma^{\prime}} \subseteq A$. This implies that $A \in \alpha O(X, \tau)_{\left[\gamma, \gamma^{\prime}\right]}$. But we have $\alpha O(X, \tau)_{\left[\gamma, \gamma^{\prime}\right]} \subseteq \alpha O(X, \tau)$. Therefore $\alpha O(X, \tau)=\alpha O(X, \tau)_{\left[\gamma, \gamma^{\prime}\right]}$.

Conversely, let $\alpha O(X, \tau)=\alpha O(X, \tau)_{\left[\gamma, \gamma^{\prime}\right]}, x \in X$ and $V$ be $\alpha$-open of $x$. Then by assumption $V$ is $\alpha_{\left[\gamma, \gamma^{\prime}\right]}$-open set. This implies that there exist $\alpha$-open sets $W$ and $S$ of $x$ such that $W^{\gamma} \cap S^{\gamma^{\prime}} \subseteq V$. Therefore $(X, \tau)$ is $\alpha_{\left[\gamma, \gamma^{\prime}\right]}$-regular.

Remark 3.27. If a space $X$ is $\alpha_{\left[\gamma, \gamma^{\prime}\right]}$-regular, then $\tau \subseteq \alpha O(X, \tau)_{\left[\gamma, \gamma^{\prime}\right]}$.

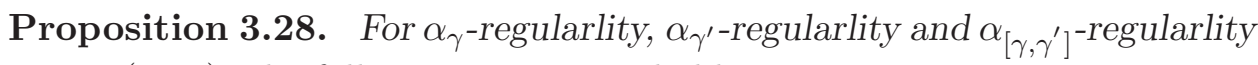
of a space $(X, \tau)$, the following properties hold.

1. $(X, \tau)$ is $\alpha_{[\gamma, X]}$-regular if and only if it is $\alpha_{\gamma}$-regular.

2. If $(X, \tau)$ is $\alpha_{\gamma}$-regular and $\alpha_{\gamma^{\prime}}$-regular, then it is $\alpha_{\left[\gamma, \gamma^{\prime}\right]}$-regular.

Definition 3.29. A subset $F$ of $(X, \tau)$ is said to be $\alpha_{\left[\gamma, \gamma^{\prime}\right]}$-closed if its complement $X \backslash F$ is $\alpha_{\left[\gamma, \gamma^{\prime}\right]^{-o p e n}}$.

We denote the set of all $\alpha_{\left[\gamma, \gamma^{\prime}\right]}$-closed sets of $(X, \tau)$ by $\alpha C(X, \tau)_{\left[\gamma, \gamma^{\prime}\right]}$.

Definition 3.30. Let $A$ be a subset of a topological space $(X, \tau)$. The intersection of all $\alpha_{\left[\gamma, \gamma^{\prime}\right]}$-closed sets containing $A$ is called the $\alpha_{\left[\gamma, \gamma^{\prime}\right]}$-closure of $A$ and denoted by $\alpha_{\left[\gamma, \gamma^{\prime}\right]^{-}} C l(A)$.

Proposition 3.31. For a point $x \in X, x \in \alpha_{\left[\gamma, \gamma^{\prime}\right]}-C l(A)$ if and only if

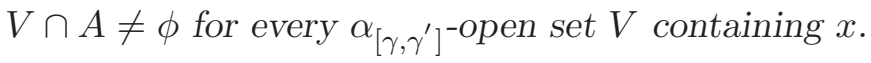

Proposition 3.32. Let $A$ and $B$ be subsets of $(X, \tau)$. Then the following hold:

1. $A \subseteq \alpha_{\left[\gamma, \gamma^{\prime}\right]^{-}} C l(A)$.

2. If $A \subseteq B$, then $\alpha_{\left[\gamma, \gamma^{\prime}\right]^{-}} C l(A) \subseteq \alpha_{\left[\gamma, \gamma^{\prime}\right]^{-}} C l(B)$. 
3. $A \in \alpha C(X, \tau)_{\left[\gamma, \gamma^{\prime}\right]}$ if and only if $\alpha_{\left[\gamma, \gamma^{\prime}\right]}-C l(A)=A$.

4. $\alpha_{\left[\gamma, \gamma^{\prime}\right]^{-}} C l(A) \in \alpha C(X, \tau)_{\left[\gamma, \gamma^{\prime}\right]}$.

5. $\alpha_{\left[\gamma, \gamma^{\prime}\right]^{-}} C l(A \cap B) \subseteq \alpha_{\left[\gamma, \gamma^{\prime}\right]}-C l(A) \cap \alpha_{\left[\gamma, \gamma^{\prime}\right]^{-}} C l(B)$.

6. If $\gamma$ and $\gamma^{\prime}$ are $\alpha$-regular, then $\alpha_{\left[\gamma, \gamma^{\prime}\right]}-C l(A \cup B)=\alpha_{\left[\gamma, \gamma^{\prime}\right]}-C l(A) \cup \alpha_{\left[\gamma, \gamma^{\prime}\right]^{-}}$ $C l(B)$.

We introduce the following definition of $\alpha C l_{\left[\gamma, \gamma^{\prime}\right]}(A)$.

Definition 3.33. For a subset $A$ of $(X, \tau)$, we define $\alpha C l_{\left[\gamma, \gamma^{\prime}\right]}(A)$ as follows: $\alpha C l_{\left[\gamma, \gamma^{\prime}\right]}(A)=\left\{x \in X:\left(U^{\gamma} \cap W^{\gamma^{\prime}}\right) \cap A \neq \phi\right.$ holds for every $\alpha$-open sets $U$ and $W$ containing $x\}$.

Remark 3.34. In Definitions 3.29, 3.30 and 3.33, put $\gamma^{\prime}=X$. Then, for any subset $A$ of $X$, the following hold:

1. $\alpha_{[\gamma, X]^{-}} C l(A)=\alpha_{\gamma^{-}} C l(A)$.

2. $\alpha C(X, \tau)_{[\gamma, X]}=\left\{F: F\right.$ is $\alpha_{\gamma}$-closed $\}$.

3. $\alpha C l_{[\gamma, X]}(A)=\alpha C l_{\gamma}(A)$.

Proposition 3.35. For a subset $A$ of $(X, \tau)$, we have $A \subseteq \alpha C l(A) \subseteq$ $\alpha C l_{\left[\gamma, \gamma^{\prime}\right]}(A) \subseteq \alpha_{\left[\gamma, \gamma^{\prime}\right]^{-}} C l(A)$.

Theorem 3.36. Let $A$ be a subset of a topological space $(X, \tau)$, the following properties are equivalent:

1. $A \in \alpha O(X, \tau)_{\left[\gamma, \gamma^{\prime}\right]}$.

2. $\alpha C l_{\left[\gamma, \gamma^{\prime}\right]}(X \backslash A)=X \backslash A$.

3. $\alpha_{\left[\gamma, \gamma^{\prime}\right]^{-}} C l(X \backslash A)=X \backslash A$.

4. $X \backslash A \in \alpha C(X, \tau)_{\left[\gamma, \gamma^{\prime}\right]}$.

Theorem 3.37. For a subset $A$ of $(X, \tau)$, the following properties hold:

1. If $(X, \tau)$ is $\alpha_{\left[\gamma, \gamma^{\prime}\right]}$-regular, then $\alpha C l(A)=\alpha C l_{\left[\gamma, \gamma^{\prime}\right]}(A)=\alpha_{\left[\gamma, \gamma^{\prime}\right]}-C l(A)$.

2. $\alpha C l_{\left[\gamma, \gamma^{\prime}\right]}(A)$ is an $\alpha$-closed subset of $(X, \tau)$. 
Theorem 3.38. Let $\gamma$ and $\gamma^{\prime}$ be $\alpha$-open operations and $A$ a subset of $(X, \tau)$. Then, the following statements hold:

1. $\alpha C l_{\left[\gamma, \gamma^{\prime}\right]}(A)=\alpha_{\left[\gamma, \gamma^{\prime}\right]^{-}} C l(A)$.

2. $\alpha C l_{\left[\gamma, \gamma^{\prime}\right]}\left(\alpha C l_{\left[\gamma, \gamma^{\prime}\right]}(A)\right)=\alpha C l_{\left[\gamma, \gamma^{\prime}\right]}(A)$.

Proof. 1. By Proposition 3.35, it suffices to prove that $\alpha_{\left[\gamma, \gamma^{\prime}\right]^{-}} C l(A) \subseteq$ $\alpha C l_{\left[\gamma, \gamma^{\prime}\right]}(A)$. Let $x \in \alpha_{\left[\gamma, \gamma^{\prime}\right]}-C l(A)$ and $W$ and $S$ be $\alpha$-open sets of $X$ containing $x$. By the $\alpha$-openness of $\gamma$ and $\gamma^{\prime}$, there exist an $\alpha_{\gamma}$-open set $W^{\prime}$ and an $\alpha_{\gamma^{\prime}}$ open set $S^{\prime}$ such that $x \in W^{\prime} \subseteq W^{\gamma}$ and $x \in S^{\prime} \subseteq S^{\gamma^{\prime}}$. By Propositions 3.17 and 3.31, $\left(S^{\prime} \cap W^{\prime}\right) \cap A \neq \phi$ and hence $\left(S^{\gamma} \cap W^{\gamma^{\prime}}\right) \cap A \neq \phi$. This implies that $x \in \alpha C l_{\left[\gamma, \gamma^{\prime}\right]}(A)$.

2. This follows immediately from (1) and Proposition 3.32 (3).

Remark 3.39. The below example shows that the equalities of Theorem 3.38 are not true without the assumption that both operations are $\alpha$-open.

Example 3.40. Let $X=\{a, b, c\}$ and $\tau=\{\phi, X,\{a\},\{b\},\{a, b\}\}$. For each $A \in \alpha O(X, \tau)$ we define two operations $\gamma$ and $\gamma^{\prime}$, respectively, by $A^{\gamma}=$ $C l(A)$ and $A^{\gamma^{\prime}}=X$. The operation $\gamma$ is not $\alpha$-open. However, $\alpha C l_{\left[\gamma, \gamma^{\prime}\right]}\{a\}=$ $\{a, c\} \subseteq \alpha_{\left[\gamma, \gamma^{\prime}\right]^{-}} C l(\{a\})=X$ and $\alpha C l_{\left[\gamma, \gamma^{\prime}\right]}\left(\alpha C l_{\left[\gamma, \gamma^{\prime}\right]}(\{a\})\right)=X \neq\{a, c\}=$ $\alpha C l_{\left[\gamma, \gamma^{\prime}\right]}(\{a\})$.

Theorem 3.41. Let $A$ and $B$ be subsets of a topological space $(X, \tau)$ and $\gamma, \gamma^{\prime}: \alpha O(X, \tau) \rightarrow P(X)$ operations on $\alpha O(X, \tau)$. Then we have the following properties:

1. $A \subseteq \alpha C l_{\left[\gamma, \gamma^{\prime}\right]}(A)$.

2. $\alpha C l_{\left[\gamma, \gamma^{\prime}\right]}(\phi)=\phi$ and $\alpha C l_{\left[\gamma, \gamma^{\prime}\right]}(X)=X$.

3. $A \in \alpha C(X, \tau)_{\left[\gamma, \gamma^{\prime}\right]}$ if and only if $\alpha C l_{\left[\gamma, \gamma^{\prime}\right]}(A)=A$.

4. If $A \subseteq B$, then $\alpha C l_{\left[\gamma, \gamma^{\prime}\right]}(A) \subseteq \alpha C l_{\left[\gamma, \gamma^{\prime}\right]}(B)$.

5. $\alpha C l_{\left[\gamma, \gamma^{\prime}\right]}(A \cup B) \subseteq \alpha C l_{\gamma}(A) \cup C l_{\alpha_{\gamma^{\prime}}}(B)$.

6. If $\gamma$ and $\gamma^{\prime}$ are $\alpha$-regular, then $\alpha C l_{\left[\gamma, \gamma^{\prime}\right]}(A \cup B)=\alpha C l_{\left[\gamma, \gamma^{\prime}\right]}(A) \cup \alpha C l_{\left[\gamma, \gamma^{\prime}\right]}(B)$. 
7. $\alpha C l_{\left[\gamma, \gamma^{\prime}\right]}(A \cap B) \subseteq \alpha C l_{\left[\gamma, \gamma^{\prime}\right]}(A) \cap \alpha C l_{\left[\gamma, \gamma^{\prime}\right]}(B)$.

Proof. (1), (2) and (4). They are obtained from Definition 3.33.

(3). Suppose that $A$ is $\alpha_{\left[\gamma, \gamma^{\prime}\right]}$-closed. Then $X \backslash A$ is $\alpha_{\left[\gamma, \gamma^{\prime}\right]^{-}}$open in $(X, \tau)$. We claim that $\alpha C l_{\left[\gamma, \gamma^{\prime}\right]}(A) \subseteq A$. Let $x \notin A$. There exist $\alpha$-open sets $U$ and $V$ of $(X, \tau)$ containing $x$ such that $U^{\gamma} \cap V^{\gamma^{\prime}} \subseteq X \backslash A$, that is, $\left(U^{\gamma} \cap V^{\gamma^{\prime}}\right) \cap A=\phi$. Hence by Definition 3.33, we have that $x \notin \alpha C l_{\left[\gamma, \gamma^{\prime}\right]}(A)$ and so $\alpha C l_{\left[\gamma, \gamma^{\prime}\right]}(A) \subseteq A$. By (1), it is proved that $\alpha C l_{\left[\gamma, \gamma^{\prime}\right]}(A)=A$. Conversely, suppose that $\alpha C l_{\left[\gamma, \gamma^{\prime}\right]}(A)=$ $A$. Let $x \in X \backslash A$. Since $x \notin \alpha C l_{\left[\gamma, \gamma^{\prime}\right]}(A)$, there exist an $\alpha$-open sets $U$ and $V$ containing $x$ such that $\left(U^{\gamma} \cap V^{\gamma^{\prime}}\right) \cap A=\phi$, that is, $U^{\gamma} \cap V^{\gamma^{\prime}} \subseteq X \backslash A$. Therefore, $A$ is $\alpha_{\left[\gamma, \gamma^{\prime}\right]^{\prime} \text { closed. }}$

(5), (7). They are obtained from (4).

(6). Let $x \notin \alpha C l_{\left[\gamma, \gamma^{\prime}\right]}(A) \cup \alpha C l_{\left[\gamma, \gamma^{\prime}\right]}(B)$. Then there exist $\alpha$-open sets $U, V, W$ and $S$ of $(X, \tau)$ containing $x$ such that $\left(U^{\gamma} \cap V^{\gamma^{\prime}}\right) \cap A=\phi$ and $\left(W^{\gamma} \cap S^{\gamma^{\prime}}\right) \cap B=\phi$. Since $\gamma$ and $\gamma^{\prime}$ are $\alpha$-regular, by definition of $\alpha$-regular, there exist $\alpha$-open sets $K$ and $L$ of $(X, \tau)$ containing $x$ such that $k^{\gamma} \subseteq U^{\gamma} \cap W^{\gamma}$ and $L^{\gamma^{\prime}} \subseteq V^{\gamma^{\prime}} \cap S^{\gamma^{\prime}}$. Thus, we have $\left(k^{\gamma} \cap L^{\gamma^{\prime}}\right) \cap(A \cup B) \subseteq\left(\left(U^{\gamma} \cap W^{\gamma}\right) \cap\left(V^{\gamma^{\prime}} \cap S^{\gamma^{\prime}}\right)\right) \cap(A \cup B)=\left(\left(U^{\gamma} \cap\right.\right.$ $\left.\left.V^{\gamma^{\prime}}\right) \cap\left(W^{\gamma} \cap S^{\gamma^{\prime}}\right)\right) \cap(A \cup B)=\left[\left(\left(U^{\gamma} \cap V^{\gamma^{\prime}}\right) \cap\left(W^{\gamma} \cap S^{\gamma^{\prime}}\right)\right) \cap A\right] \cup\left[\left(\left(U^{\gamma} \cap V^{\gamma^{\prime}}\right) \cap\left(W^{\gamma} \cap\right.\right.\right.$ $\left.\left.\left.S^{\gamma}\right)\right) \cap B\right]=\phi$, that is, $\left(k^{\gamma} \cap L^{\gamma}\right) \cap(A \cup B)=\phi$. Hence, $x \notin \alpha C l_{\left[\gamma, \gamma^{\prime}\right]}(A \cup B)$. This shows that $\alpha C l_{\left[\gamma, \gamma^{\prime}\right]}(A) \cup \alpha C l_{\left[\gamma, \gamma^{\prime}\right]}(B) \supseteq \alpha C l_{\left[\gamma, \gamma^{\prime}\right]}(A \cup B)$.

Remark 3.42. Example 3.3 shows that the inclusion of Theorem 3.41 (5) is a proper one in general. For a subset $\{a\}, \alpha C l_{\left[\gamma, \gamma^{\prime}\right]}(\{a\})=\{a\} \subseteq \alpha C l_{\gamma}(\{a\}) \cup$ $\alpha C l_{\gamma^{\prime}}(\{a\})=\{a, b\}$.

We define the $\alpha_{\left[\gamma, \gamma^{\prime}\right]}$-interior of a subset $A$ of $(X, \tau)$ as follows:

Definition 3.43. Let $A$ be a subset of a topological space $(X, \tau)$. The union of all $\alpha_{\left[\gamma, \gamma^{\prime}\right]^{-}}$-open sets contained in $A$ is called the $\alpha_{\left[\gamma, \gamma^{\prime}\right]^{\prime}}$-interior of $A$ and is denoted by $\alpha_{\left[\gamma, \gamma^{\prime}\right]^{-}} \operatorname{Int}(A)$.

Proposition 3.44. For any subsets $A, B$ of $X$, we have the following:

1. $\alpha_{\left[\gamma, \gamma^{\prime}\right]^{\prime}} \operatorname{Int}(A)$ is an $\alpha_{\left[\gamma, \gamma^{\prime}\right]}$-open set in $X$.

2. $A$ is $\alpha_{\left[\gamma, \gamma^{\prime}\right]}$-open if and only if $A=\alpha_{\left[\gamma, \gamma^{\prime}\right]}-\operatorname{Int}(A)$.

3. $\alpha_{\left[\gamma, \gamma^{\prime}\right]^{-}} \operatorname{Int}\left(\alpha_{\left[\gamma, \gamma^{\prime}\right]^{\prime}} \operatorname{Int}(A)\right)=\alpha_{\left[\gamma, \gamma^{\prime}\right]^{-} \operatorname{Int}(A)}$.

4. $\alpha_{\left[\gamma, \gamma^{\prime}\right]}-\operatorname{Int}(A) \subseteq A$. 
5. If $A \subseteq B$, then $\alpha_{\left[\gamma, \gamma^{\prime}\right]}-\operatorname{Int}(A) \subseteq \alpha_{\left[\gamma, \gamma^{\prime}\right]}-\operatorname{Int}(B)$.

6. $\alpha_{\left[\gamma, \gamma^{\prime}\right]}-\operatorname{Int}(A \cup B) \supseteq \alpha_{\left[\gamma, \gamma^{\prime}\right]}-\operatorname{Int}(A) \cup \alpha_{\left[\gamma, \gamma^{\prime}\right]}-\operatorname{Int}(B)$.

7. $\alpha_{\left[\gamma, \gamma^{\prime}\right]}-\operatorname{Int}(A \cap B) \subseteq \alpha_{\left[\gamma, \gamma^{\prime}\right]}-\operatorname{Int}(A) \cap \alpha_{\left[\gamma, \gamma^{\prime}\right]}-\operatorname{Int}(B)$.

Proof. Obvious.

Proposition 3.45. Let $A$ be any subset of a topological space $(X, \tau)$. Then the following statements are true:

1. $X \backslash \alpha_{\left[\gamma, \gamma^{\prime}\right]^{-}} \operatorname{Int}(A)=\alpha_{\left[\gamma, \gamma^{\prime}\right]}-C l(X \backslash A)$.

2. $X \backslash \alpha_{\left[\gamma, \gamma^{\prime}\right]}-C l(A)=\alpha_{\left[\gamma, \gamma^{\prime}\right]}-\operatorname{Int}(X \backslash A)$.

3. $\alpha_{\left[\gamma, \gamma^{\prime}\right]}-\operatorname{Int}(A)=X \backslash \alpha_{\left[\gamma, \gamma^{\prime}\right]}-C l(X \backslash A)$.

4. $\alpha_{\left[\gamma, \gamma^{\prime}\right]}-C l(A)=X \backslash \alpha_{\left[\gamma, \gamma^{\prime}\right]}-\operatorname{Int}(X \backslash A)$.

\section{4. $\left(\alpha_{\left[\gamma, \gamma^{\prime}\right]}, \alpha_{\left[\beta, \beta^{\prime}\right]}\right)$-Functions}

Throughout this section, let $f:(X, \tau) \rightarrow(Y, \sigma)$ be a function and $\gamma, \gamma^{\prime}$ : $\alpha O(X, \tau) \rightarrow P(X)$ be operations on $\alpha O(X, \tau)$ and $\beta, \beta^{\prime}: \alpha O(Y, \sigma) \rightarrow P(Y)$ be operations on $\alpha O(Y, \sigma)$.

Definition 4.1. A function $f:(X, \tau) \rightarrow(Y, \sigma)$ is said to be $\left(\alpha_{\left[\gamma, \gamma^{\prime}\right]}\right.$, $\left.\alpha_{\left[\beta, \beta^{\prime}\right]}\right)$-continuous if for each point $x \in X$ and each $\alpha$-open sets $W$ and $S$ of $(Y, \sigma)$ containing $f(x)$ there exist $\alpha$-open sets $U$ and $V$ of $(X, \tau)$ containing $x$ such that $f\left(U^{\gamma} \cap V^{\gamma^{\prime}}\right) \subseteq W^{\beta} \cap S^{\beta^{\prime}}$.

Theorem 4.2. Let (1), (2), (3), (4), (5), (6) and (7) be the following properties for a function $f:(X, \tau) \rightarrow(Y, \sigma)$.

1. $f:(X, \tau) \rightarrow(Y, \sigma)$ is $\left(\alpha_{\left[\gamma, \gamma^{\prime}\right]}, \alpha_{\left[\beta, \beta^{\prime}\right]}\right)$-continuous.

2. $f\left(\alpha C l_{\left[\gamma, \gamma^{\prime}\right]}(A)\right) \subseteq \alpha C l_{\left[\beta, \beta^{\prime}\right]}(f(A))$ for every subset $A$ of $(X, \tau)$.

3. $\alpha C l_{\left[\gamma, \gamma^{\prime}\right]}\left(f^{-1}(B)\right) \subseteq f^{-1}\left(\alpha C l_{\left[\beta, \beta^{\prime}\right]}(B)\right)$ for every subset $B$ of $(Y, \sigma)$.

4. $f^{-1}(B)$ is $\alpha_{\left[\gamma, \gamma^{\prime}\right]}$-closed for every $\alpha_{\left[\beta, \beta^{\prime}\right]}$-closed set $B$ of $(Y, \sigma)$.

5. $f\left(\alpha_{\left[\gamma, \gamma^{\prime}\right]^{-}} C l(A)\right) \subseteq \alpha_{\left[\beta, \beta^{\prime}\right]^{-}} C l(f(A))$ for every subset $A$ of $(X, \tau)$. 


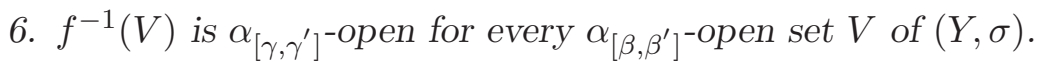

7. for each point $x \in X$ and each $\alpha_{\left[\beta, \beta^{\prime}\right]}$-open $W$ of $(Y, \sigma)$ containing $f(x)$ there exist $\alpha_{\left[\gamma, \gamma^{\prime}\right]}$-open $U$ of $(X, \tau)$ containing $x$ such that $f(U) \subseteq W$.

Then $(1) \Rightarrow(2) \Leftrightarrow(3) \Rightarrow(4) \Leftrightarrow(5) \Leftrightarrow(6) \Leftrightarrow(7)$ hold.

Proof. (1) $\Rightarrow(2)$. Let $x \in \alpha C l_{\left[\gamma, \gamma^{\prime}\right]}(A)$ and $W, S$ be $\alpha$-open sets of $(Y, \sigma)$ containing $f(x)$. There exist $\alpha$-open sets $U$ and $V$ of $(X, \tau)$ containing $x$ such that $f\left(U^{\gamma} \cap V^{\gamma^{\prime}}\right) \subseteq W^{\beta} \cap S^{\beta^{\prime}}$. Since $x \in \alpha C l_{\left[\gamma, \gamma^{\prime}\right]}(A)$, then $\left(U^{\gamma} \cap V^{\gamma^{\prime}}\right) \cap A \neq \phi$, implies that $f\left(U^{\gamma} \cap V^{\gamma^{\prime}}\right) \cap f(A) \neq \phi$. Therefore, we have $f(A) \cap\left(W^{\beta} \cap S^{\beta^{\prime}}\right) \neq \phi$. Therefore $f(x) \in \alpha C l_{\left[\beta, \beta^{\prime}\right]}(f(A))$, which implies that $x \in f^{-1}\left(\alpha C l_{\left[\beta, \beta^{\prime}\right]}(f(A))\right)$. Hence $\alpha C l_{\left[\gamma, \gamma^{\prime}\right]}(A) \subseteq f^{-1}\left(\alpha C l_{\left[\beta, \beta^{\prime}\right]}(f(A))\right)$, so that $f\left(\alpha C l_{\left[\gamma, \gamma^{\prime}\right]}(A)\right) \subseteq \alpha C l_{\left[\beta, \beta^{\prime}\right]}(f(A))$.

$(2) \Rightarrow(3)$. Let $B$ be any subset of $Y$. Then $f^{-1}(B)$ is a subset of $X$. By (2), we have $f\left(\alpha C l_{\left[\gamma, \gamma^{\prime}\right]}\left(f^{-1}(B)\right)\right) \subseteq \alpha C l_{\left[\beta, \beta^{\prime}\right]}\left(f\left(f^{-1}(B)\right)\right) \subseteq \alpha C l_{\left[\beta, \beta^{\prime}\right]}(B)$. Hence $\alpha C l_{\left[\gamma, \gamma^{\prime}\right]}\left(f^{-1}(B)\right) \subseteq f^{-1}\left(\alpha C l_{\left[\beta, \beta^{\prime}\right]}(B)\right)$.

$(3) \Rightarrow(2)$. Let $A$ be any subset of $X$. Then $f(A)$ is a subset of $Y$. By (3), we have $\alpha C l_{\left[\gamma, \gamma^{\prime}\right]}\left(f^{-1} f((A))\right) \subseteq f^{-1}\left(\alpha C l_{\left[\beta, \beta^{\prime}\right]}(f(A))\right)$. This implies that $\alpha C l_{\left[\gamma, \gamma^{\prime}\right]}(A) \subseteq f^{-1}\left(\alpha C l_{\left[\beta, \beta^{\prime}\right]}(f(A))\right)$. Hence $f\left(\alpha C l_{\left[\gamma, \gamma^{\prime}\right]}(A)\right) \subseteq \alpha C l_{\left[\beta, \beta^{\prime}\right]}(f(A))$.

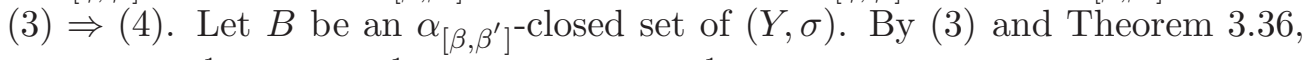
$\alpha C l_{\left[\gamma, \gamma^{\prime}\right]}\left(f^{-1}(B)\right) \subseteq f^{-1}(B)$ and hence $f^{-1}(B)$ is $\alpha_{\left[\gamma, \gamma^{\prime}\right]}$-closed.

$(4) \Rightarrow(5)$. Let $A$ be any subset of $X$. Then $f(A) \subseteq \alpha_{\left[\beta, \beta^{\prime}\right]^{-}} C l(f(A))$ and $\alpha_{\left[\beta, \beta^{\prime}\right]^{-}}$ $C l(f(A))$ is an $\alpha_{\left[\beta, \beta^{\prime}\right]^{-}}$-closed set in $Y$. Hence $A \subseteq f^{-1}\left(\alpha_{\left[\beta, \beta^{\prime}\right]^{-}} C l(f(A))\right)$. By (4), we have $f^{-1}\left(\alpha_{\left[\beta, \beta^{\prime}\right]^{-}} C l(f(A))\right)$ which is an $\alpha_{\left[\gamma, \gamma^{\prime}\right]^{-c l o s e d}}$ set in $X$. Therefore, $\alpha_{\left[\gamma, \gamma^{\prime}\right]^{-}} C l(A) \subseteq f^{-1}\left(\alpha_{\left[\beta, \beta^{\prime}\right]^{-}} C l(f(A))\right)$. Hence $f\left(\alpha_{\left[\gamma, \gamma^{\prime}\right]^{-}} C l(A)\right) \subseteq \alpha_{\left[\beta, \beta^{\prime}\right]^{-}}$ $C l(f(A))$.

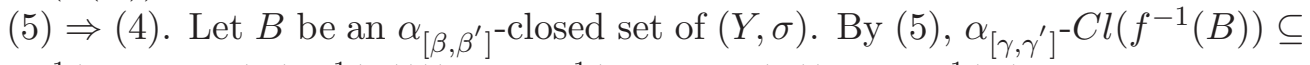
$f^{-1}\left(\alpha_{\left[\beta, \beta^{\prime}\right]^{-}} C l\left(f\left(f^{-1}(B)\right)\right)\right) \subseteq f^{-1}\left(\alpha_{\left[\beta, \beta^{\prime}\right]^{-}} C l(B)\right) \subseteq f^{-1}(B)$. Therefore, by Proposition 3.32, $f^{-1}(B)$ is $\alpha_{\left[\gamma, \gamma^{\prime}\right]^{-}}$-closed.

$(5) \Leftrightarrow(6)$. This follows from Definition 3.29 and the equivalence of $(4) \Leftrightarrow(5)$.

$(6) \Rightarrow(7)$. Let $W$ be any $\alpha_{\left[\beta, \beta^{\prime}\right]^{-}}$open set in $Y$ containing $f(x)$, so its in-

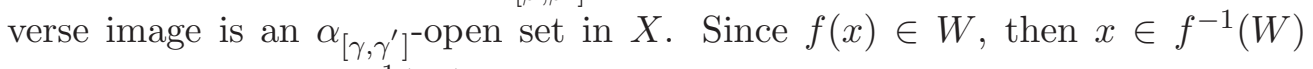
and by hypothesis $f^{-1}(W)$ is an $\alpha_{\left[\gamma, \gamma^{\prime}\right]}$-open set in $X$ containing $x$ so that $f\left(f^{-1}(W)\right) \subseteq W$.

$(7) \Rightarrow(6)$. Let $V \in \alpha O(Y, \sigma)_{\left[\beta, \beta^{\prime}\right]}$. For each $x \in f^{-1}(V)$, by (7), there exists an $\alpha_{\left[\gamma, \gamma^{\prime}\right]}$-open set $U_{x}$ containing $x$ such that $f\left(U_{x}\right) \subseteq V$. Then we have $f^{-1}(V)=$ 
$\cup\left\{U_{x} \in \alpha O(X, \tau)_{\left[\gamma, \gamma^{\prime}\right]}: x \in f^{-1}(V)\right\}$ and hence $f^{-1}(V) \in \alpha O(X, \tau)_{\left[\gamma, \gamma^{\prime}\right]}$ using Proposition 3.2.

Corollary 4.3. If $(Y, \sigma)$ is an $\alpha_{\left[\beta, \beta^{\prime}\right]}$-regular space, or operations $\beta$ and $\beta^{\prime}$ are $\alpha$-open on $\alpha O(Y, \sigma)$, then all properties of Theorem 4.2 are equivalent.

Proof. By Theorem 4.2, it is sufficient to prove the implication $(4) \Rightarrow(1)$, where (1) and (4) are the properties of Theorem 4.2.

First, we show the implication under the assumption that $(Y, \sigma)$ is an $\alpha_{\left[\beta, \beta^{\prime}\right]^{-}}$ regular space. Let $x \in X$ and $W, S$ be $\alpha$-open sets of $(Y, \sigma)$ containing $f(x)$. By Proposition 3.26, $Y \backslash(W \cap S)$ is $\alpha_{\left[\beta, \beta^{\prime}\right]}$-closed. Then, $f^{-1}(Y \backslash(W \cap S))$ is

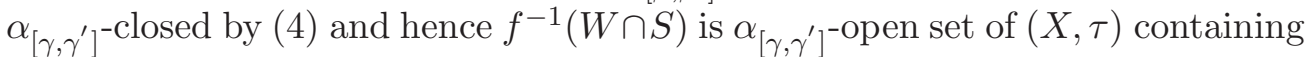
$x$. Therefore, there exist $\alpha$-open sets $U$ and $V$ of $(X, \tau)$ containing $x$ such that $U^{\gamma} \cap V^{\gamma^{\prime}} \subseteq f^{-1}(W \cap S)$ and so $f\left(U^{\gamma} \cap V^{\gamma^{\prime}}\right) \subseteq W^{\beta} \cap S^{\beta^{\prime}}$. This implies that $f$ is $\left(\alpha_{\left[\gamma, \gamma^{\prime}\right]}, \alpha_{\left[\beta, \beta^{\prime}\right]}\right)$-continuous.

Second, we suppose that the operations $\beta$ and $\beta^{\prime}$ are $\alpha$-open. Let $x \in X$ and $W, S$ be $\alpha$-open sets of $(Y, \sigma)$ containing $f(x)$. By using $\alpha$-openness of $\beta$ and

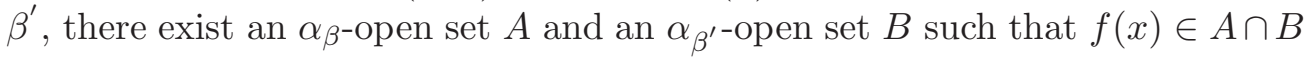
and $A \cap B \subseteq W^{\beta} \cap S^{\beta^{\prime}}$. By Proposition 3.17, $Y \backslash(A \cap B)$ is $\alpha_{\left[\gamma, \gamma^{\prime}\right]}$-closed

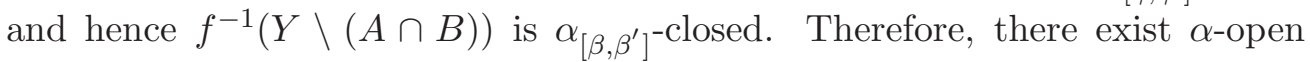
sets $U$ and $V$ of $(X, \tau)$ containing $x$ such that $U^{\gamma} \cap V^{\gamma^{\prime}} \subseteq f^{-1}(A \cap B)$ and so $f\left(U^{\gamma} \cap V^{\gamma^{\prime}}\right) \subseteq W^{\beta} \cap S^{\beta^{\prime}}$. This implies that $f$ is $\left(\alpha_{\left[\gamma, \gamma^{\prime}\right]}, \alpha_{\left[\beta, \beta^{\prime}\right]}\right)$-continuous.

Remark 4.4. It is clear that the identity operation and the operation $X: \alpha O(X, \tau) \rightarrow(X, \tau)$ are $\alpha$-open on $\alpha O(X, \tau)$. Therefore, by Corollary 4.3, if $\beta$ and $\beta^{\prime}$ are chosen from mentioned operations above, then all properties of Theorem 4.2 are equivalent.

Remark 4.5. The converse of implication $(1) \Rightarrow(4)$ in Theorem 4.2 is not true in general as shown by the following example.

Example 4.6. Let $X=\{1,2,3\}$ and $\tau=\{\phi, X,\{1\},\{2\},\{1,2\}\}$ be a topology on $X$. Let $f:(X, \tau) \rightarrow(X, \tau)$ be the identity. Let $\gamma=\gamma^{\prime}=\beta^{\prime}=X$ : $\alpha O(X, \tau) \rightarrow P(X)$ be the operations on $\alpha O(X, \tau)$ and $\beta$ the closure operation on $\alpha O(X, \tau)$. Then, the condition (4) in Theorem 4.2 is true. It is shown that $f$ is not $\left(\alpha_{\left[\gamma, \gamma^{\prime}\right]}, \alpha_{\left[\beta, \beta^{\prime}\right]}\right)$-continuous.

Theorem 4.2 suggests the following.

Remark 4.7. If $f:(X, \tau) \rightarrow(Y, \sigma)$ is $\left(\alpha_{\left[\gamma, \gamma^{\prime}\right]}, \alpha_{\left[\beta, \beta^{\prime}\right]}\right)$-continuous, then the induced function $f:\left(X, \alpha O(X, \tau)_{\left[\gamma, \gamma^{\prime}\right]}\right) \rightarrow\left(Y, \alpha O(Y, \sigma)_{\left[\beta, \beta^{\prime}\right]}\right)$ is continuous. 
Remark 4.8. The converse of Remark 4.7 is not true in general as shown by the following example.

Example 4.9. Let $(X, \tau)$ as in Example 4.6 and $f:(X, \tau) \rightarrow(X, \tau)$ be a function defined by

$$
f(x)=\left\{\begin{array}{cc}
2 & \text { if } x=1 \\
3 & \text { if } x=2 \\
1 & \text { if } x=3
\end{array}\right.
$$

moreover let $\gamma=\beta$ be the closure operation on $\alpha O(X, \tau)$ and $\gamma^{\prime}=\beta^{\prime}=$ $X: \alpha O(X, \tau) \rightarrow P(X)$. Then, $\alpha O(X, \tau)_{[\gamma, X]}=\{\phi, X\}$ and it is shown that $f$ is not $\left(\alpha_{[\gamma, X]}, \alpha_{[\gamma, X]}\right)$-continuous. However, $f:\left(X, \alpha O(X, \tau)_{\left[\gamma, \gamma^{\prime}\right]}\right) \rightarrow$ $\left(Y, \alpha O(Y, \sigma)_{\left[\beta, \beta^{\prime}\right]}\right)$ is continuous.

Let $(X, \tau),(Y, \sigma)$ and $(Z, \eta)$ be spaces and $\gamma, \gamma^{\prime}: \alpha O(X, \tau) \rightarrow P(X)$, $\beta, \beta^{\prime}: \alpha O(Y) \rightarrow P(Y)$ and $\delta, \delta^{\prime}: \alpha O(Z) \rightarrow P(Z)$, be operations on $\alpha O(X, \tau)$, $\alpha O(Y, \sigma)$ and $\alpha O(Z, \eta)$, respectively.

Theorem 4.10. If $f:(X, \tau) \rightarrow(Y, \sigma)$ is $\left(\alpha_{\left[\gamma, \gamma^{\prime}\right]}, \alpha_{\left[\beta, \beta^{\prime}\right]}\right)$-continuous and $g:(Y, \sigma) \rightarrow(Z, \eta)$ is $\left(\alpha_{\left[\beta, \beta^{\prime}\right]}, \alpha_{\left[\delta, \delta^{\prime}\right]}\right)$-continuous, then its composition gof : $(X, \tau) \rightarrow(Z, \eta)$ is $\left(\alpha_{\left[\gamma, \gamma^{\prime}\right]}, \alpha_{\left[\delta, \delta^{\prime}\right]}\right)$-continuous.

Proof. Let $x \in X, K$ and $L$ be $\alpha$-open sets of $Z$ containing $g(f(x))$. Since $g$ is $\left(\alpha_{\left[\beta, \beta^{\prime}\right]}, \alpha_{\left[\delta, \delta^{\prime}\right]}\right)$-continuous, then there exist $\alpha$-open sets $W$ and $S$ of $Y$ containing $f(x)$ such that $g\left(W^{\beta} \cap S^{\beta^{\prime}}\right) \subseteq K^{\delta} \cap L^{\delta^{\prime}}$. Also, since $f$ is $\left(\alpha_{\left[\gamma, \gamma^{\prime}\right]}\right.$, $\left.\alpha_{\left[\beta, \beta^{\prime}\right]}\right)$-continuous, then there exist $\alpha$-open sets $U$ and $V$ of $X$ containing $x$ such that $f\left(U^{\gamma} \cap V^{\gamma^{\prime}}\right) \subseteq W^{\beta} \cap S^{\beta^{\prime}}$. This implies that $f\left(U^{\gamma} \cap V^{\gamma^{\prime}}\right) \subseteq W^{\beta} \cap S^{\beta^{\prime}} \subseteq$ $g^{-1}\left(K^{\delta} \cap L^{\delta^{\prime}}\right)$. Then we obtain $(g \circ f)\left(U^{\gamma} \cap V^{\gamma^{\prime}}\right) \subseteq K^{\delta} \cap L^{\delta^{\prime}}$. Therefore, gof is $\left(\alpha_{\left[\gamma, \gamma^{\prime}\right]}, \alpha_{\left[\delta, \delta^{\prime}\right]}\right)$-continuous.

Definition 4.11. A function $f:(X, \tau) \rightarrow(Y, \sigma)$ is said to be $\left(\alpha_{\left[\gamma, \gamma^{\prime}\right]}\right.$,

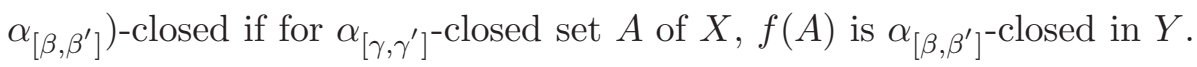

Proposition 4.12. Let $f:(X, \tau) \rightarrow(Y, \sigma)$ be an $\left(\alpha_{\left[\gamma, \gamma^{\prime}\right]}, \alpha_{\left[\beta, \beta^{\prime}\right]}\right)$-closed function. Then, for each subset $B$ of $(Y, \sigma)$ and each $\alpha_{\left[\gamma, \gamma^{\prime}\right]}$-open set $U$ containing $f^{-1}(B)$, there exists an $\alpha_{\left[\beta, \beta^{\prime}\right]}$-open set $V$ such that $B \subseteq V$ and $f^{-1}(V) \subseteq U$.

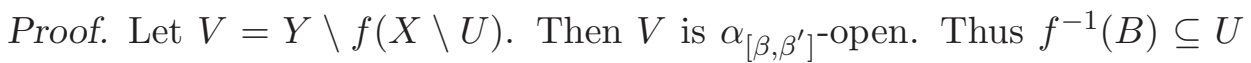
implies $B \subseteq V$ and $f^{-1}(V)=f^{-1}(Y \backslash f(X \backslash U))=X \backslash f^{-1}(f(X \backslash U)) \subseteq$ $X \backslash(X \backslash U)=U$, or $f^{-1}(V) \subseteq U$. 
Proposition 4.13. If $f:(X, \tau) \rightarrow(Y, \sigma)$ is bijective and $f^{-1}:(Y, \sigma) \rightarrow$ $(X, \tau)$ is $\left(\alpha_{\left[\beta, \beta^{\prime}\right]}, \alpha_{\left[\gamma, \gamma^{\prime}\right]}\right)$-continuous, then $f$ is $\left(\alpha_{\left[\gamma, \gamma^{\prime}\right]}, \alpha_{\left[\beta, \beta^{\prime}\right]}\right)$-closed.

Proof. This follows from definitions and Theorem 4.2.

\section{References}

[1] A. S. Mashhour, A. A. Allam, F. S. Mahmoud and F. H. Khedr, On supra topological spaces, Indian J. Pure and Appl. Math., 14, No. 4 (1983), 502-510.

[2] S. Kasahara, Operation-compact spaces, Math. Japonica, 24 (1979), 97105.

[3] O. Njastad, On some classes of nearly open sets, Pacific J. Math., 15 (1965), 961-970.

[4] H. Ogata, Operation on topological spaces and associated topology, Math. Japonica, 36, No. 1 (1991), 175-184.

[5] H. Maki and T. Noiri, Bioperations and some separation axioms, Scientiae Mathematicae Japonicae Online, 4 (2001), 165-180.

[6] H. Z. Ibrahim, On a class of $\alpha_{\gamma}$-open sets in a topological space, Acta Scientiarum. Technology, 35, No. 3 (2013), 539-545.

[7] J. Umehara, H. Maki and T. Noiri, Bioperations on topological spaces and some separation axioms, Mem. Fac. Sci. Kochi Univ. Ser. A (Math.), 13 (1992), 45-59. 